\title{
Eventos de vida en la infancia y su relación con el trastorno de estrés postraumático en adultos en Lima Metropolitana.
}

Childhood life events and their relationship with post-traumatic stress disorder in adults in Metropolitan Lima.

\author{
Christian Portella 1,a, Diana Mayo-Arpi 1,b, Nathanael Valladares-Lujan 1,c Javier E. Saavedra 1,2,d
}

\section{RESUMEN}

Objetivos: Determinar la relación entre los eventos de vida en la infancia y el trastorno de estrés postraumático (TEPT) en la población adulta de Lima Metropolitana. Materiales y métodos: Se realizó un análisis secundario del Estudio Epidemiológico de Salud Mental de Lima Metropolitana 2 012, que utilizó los instrumentos de la versión breve y modificada del Egna Minen av Bardoms Uppfostran (EMBU), cuestionario de eventos adversos tempranos y Mini Entrevista Neuropsiquiátrica Internacional (MINI), versión española. Resultados: Aquellos que recibieron una crianza más permisiva para con actividades no permitidas a sus hermanos(as) presentaron una relación estadísticamente significativa con el TEPT (OR 1,70, IC 95\% 1,09-2,65, p=0,019, al igual que eventos vitales tales como haber sido objeto o haber sufrido de chantajes, manipulaciones o humillaciones (OR 6,22, IC 95\% 4,11-9,42, p <0,001). Conclusiones: Existe alguna relación entre estilo parental de crianza permisiva ("Le permitían hacer cosas que a sus hermanos(as) no les permitían") y experiencias de eventos adversos ("Mientras crecía fue objeto (o sufrió) de chantajes, manipulaciones o humillaciones en forma frecuente") en la infancia, con el TEPT en habitantes adultos de Lima Metropolitana.

PALABRAS CLAVE: Trastorno por estrés postraumático, adultos sobrevivientes de eventos adversos infantiles, crianza del niño.

\section{SUMMARY}

Objectives: To determine the relationship between childhood life events and PTSD in adult population of Metropolitan Lima. Materials and methods: A secondary analysis was carried out of the Epidemiological Study of Mental Health of Metropolitan Lima 2 012, which used the instruments of a short and modified version of the Egna Minen av Bardoms Uppfostran (EMBU), a questionnaire on early adverse events, and the Mini Internacional Neuropsychiatric Interview (MINI), Spanish version. Results: Those who received a more permissive upbringing being allowed to do activities that their siblings were not, presented a statistically significant relationship with PTSD (OR 1.70, $95 \%$ CI 1.09-2.65, $\mathrm{p}=0.019)$, the same as those who experienced adverse life events or were the object of or had suffered blackmail, manipulations or humiliations (OR 6.22, 95\% CI 4.11-9.42, p $<0.001$ ). Conclusions: There is a

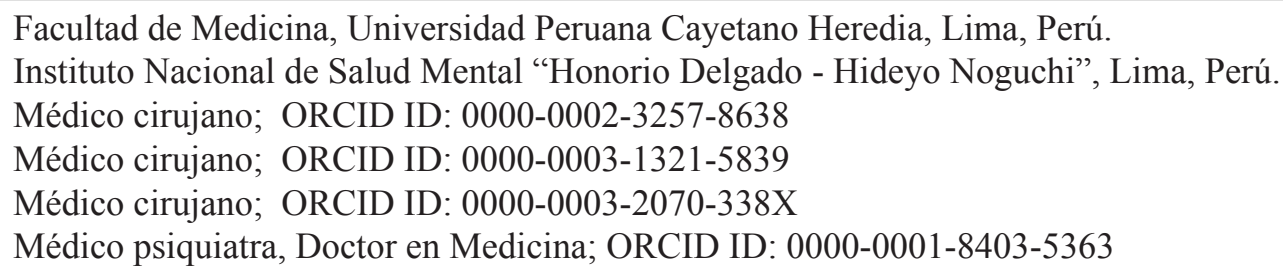


Eventos de vida en la infancia y su relación con el trastorno de estrés postraumático en adultos en Lima Metropolitana.

relationship between permissive parenting style ("They allowed him to do things that his siblings were not allowed to do"), and the experience of childhood adverse events ("While growing up, he was subjected to (or suffered) from frequent blackmail, manipulation or humiliations)" with PTSD, in adults from Metropolitan Lima.

KEYWORDS: Post-traumatic stress disorder, adult survivors of child adverse events, child rearing.

\section{INTRODUCCIÓN}

El trastorno de estrés postraumático (TEPT), es un trastorno psiquiátrico, "que surge como respuesta tardía a un estresor, y que causa malestar generalizado en casi todo el mundo. Tiene un periodo de latencia que puede durar desde unas pocas semanas hasta meses, superando rara vez los 6 meses. Pese a que se espera la recuperación en la mayoría de los casos, en una pequeña porción se puede tornar crónico" (1). Empero no todos los individuos que afrontan la misma experiencia traumática llegan a presentar este trastorno (2), lo cual sugiere otros factores de riesgo que podrían predisponer su aparición.

La prevalencia del TEPT varía significativamente entre cada estudio, evento y país; y puede oscilar entre 1 a $9 \%$ de prevalencia de vida de TEPT en países desarrollados $(3,4,5)$, como también, en países latinoamericanos oscila entre 4,4 a $11,2 \%$ $(6,7,8)$. En Perú, la prevalencia también varía según las regiones; por ejemplo, en Lima (2012) se encontró una prevalencia de $5,1 \%$ (8); en cambio, en otras poblaciones como en áreas circundantes a las ciudades de Iquitos y Pucallpa (2009), se encontró una prevalencia de TEPT de 7,8\% (9).

A nuestro entender, hay pocos estudios sobre TEPT en Latinoamérica; y el estudio de los desórdenes de ansiedad en nuestra región resulta importante debido al efecto cultural, a diferencia de países desarrollados (10), Estos estudios se han enfocado en eventos específicos; sin embargo, no han estudiado otros acontecimientos positivos $\mathrm{y} / \mathrm{o}$ negativos que tuvieran impacto en la vida del individuo y una relación con el TEPT. En el caso del Perú, la mayoría de los estudio corresponden a tasas de prevalencia luego de un suceso catastrófico $(11,12)$.

Los factores de riesgo que predisponen a padecer un TEPT se han estudiado en diferentes poblaciones. Ozer et al., (13) y Brewin et al., (14) realizaron revisiones sistemáticas sobre los factores de riesgo de TEPT, los cuales los dividieron en factores pretrauma, trauma y postrauma. El estudio de Ozer et al., reportó que los factores peritraumáticos, como la respuesta emocional peritraumática y la disociación peritraumática, eran los mayores predictores de TEPT (13); y, en el de Brewin et al se encontró que los factores del trauma (disociación y respuestas de afrontamiento) y postrauma (síntomas emergentes y apoyo social) eran los que mayor efecto predictivo tenían para padecer TEPT (14).

La infancia es una etapa de transición, definida como un periodo separado de la edad adulta, en que la persona debe estar rodeada de un ambiente de protección y cuidado para poder tener un desarrollo adecuado (15). Existen varios estudios que mencionan la relación entre las experiencias de la infancia y el TEPT. Por ejemplo, Bremner et al investigaron la frecuencia de abuso infantil en 38 veteranos de guerra con TEPT y 28 sin TEPT, encontrando una asociación significativa entre antecedente de abuso infantil y TEPT (26\% vs 7\%) (16). Así mismo, Breslau et al investigaron la influencia de traumas previos y TEPT en 2181 veteranos, encontrando una mayor predisposición a desarrollar TEPT en la adultez en personas que fueron víctimas de maltrato antes de los 15 años (17).

La mayor parte de estos estudios han dejado de lado a los eventos de vida y estilos de crianza en la infancia que podrían llegar a tener relación con el TEPT, como pueden ser las características familiares, imposiciones, favoritismo en la familia; por lo cual, en este estudio, se enfocó en los siguientes objetivos: 1) Determinar la relación entre los eventos de vida en la infancia y el TEPT en la población adulta de Lima Metropolitana, 2) Estimar los factores sociodemográficos relacionados al TEPT en la población adulta de Lima Metropolitana, 3) Estimar la relación entre los diferentes estilos de crianza recibidos en la infancia y la presencia del TEPT en la población adulta de Lima Metropolitana, 4) Estimar la relación entre algunos eventos adversos de vida tempranos y el TEPT en la población adulta de Lima Metropolitana, y 5) Determinar la relación entre los eventos de vida en la infancia y el TEPT en la población desde el punto de vista de género. 


\section{MATERIAL Y MÉTODOS}

\section{Diseño de estudio}

Este es un estudio secundario de tipo transversal analítico que utilizó la base de datos del Estudio Epidemiológico de Salud Mental de Lima Metropolitana y Callao (EESMLMC), realizado por el Instituto Nacional de Salud Mental Honorio Delgado - Hideyo Noguchi (INSM HD-HN), una encuesta realizada el 2012 con una muestra representativa de personas de Lima Metropolitana y Callao, seleccionada a través de un procedimiento probabilístico trietápico (3).

\section{Población}

La población del presente estudio incluyó a personas a partir de los 18 años residentes en viviendas particulares de Lima Metropolitana y Callao 2012.

\section{Definición de la población en estudio}

1. Características generales:

- Criterios de inclusión:

- $\quad$ Edad a partir de los 18 años, ambos sexos.

- Residentes en viviendas particulares de Lima Metropolitana y Callao durante la encuesta realizada del estudio original en el 2012.

- Criterios de exclusión:

- Personas con problemas para la comunicación verbal.

- Personas con residencia en la ciudad menor a un mes.

2. Ubicación en el espacio y tiempo:

- La encuesta del estudio original se desarrolló de mayo a diciembre del 2012.

\section{Diseño muestral:}

Unidad de análisis:

El estudio original contempló 4 unidades de análisis: adultos, adolescentes, mujeres unidas jefas de hogar o esposas del jefe de hogar, y adultos mayores; en el que cada hogar objetivo se logre entrevistar a una persona por cada unidad de análisis. Nuestro estudio se centrará en la unidad de análisis del adulto de 18 años o más.

\section{Muestra}

Este estudio toma la muestra representativa de los adultos encuestados en el EESMLMC replicación 2012. Dicha investigación logró recolectar la información de 4445 adultos (8).

\section{Procedimientos y técnicas}

\section{Instrumentos:}

Se han validado los instrumentos en su totalidad en estudios previos hechos por el INSM HD-HN (18), estas son:

- La escala de propia memoria acerca de la crianza o Egna Minen av Bardoms Uppfostran (EMBU) versión modificada y resumida: se creó una versión reducida de trece preguntas en investigaciones anteriores del INSM HD - HN, además de agregar otras cinco sobre control sin afecto, sobreprotección, autonomía personal y favoritismo machista (19). El análisis psicométrico de estas 18 preguntas reportada por el INSM HD - HN fue de una consistencia interna según el alfa de Cronbach de 0,74; se identificaron tres dimensiones: sobreprotección y exigencias, favoritismo con respecto a los hermanos y actitudes afectivas (de control y rechazo, o calor emocional); en cuanto a las actitudes afectivas, las variables de control y rechazo, también llamadas estilos afectivos negativos, son aquellos estilos de crianza que afectan negativamente en el desarrollo del niño, y las variables de calor emocional, también llamadas estilos afectivos positivos, son aquellos estilos de crianza que apoyan en el desarrollo del niño, siendo la ausencia la que se buscará asociación con el TEPT (18).

- Mini Entrevista Neuropsiquiátrica Internacional (MINI) versión española CIE-10: Basado en los criterios diagnósticos de la CIE-10, es un instrumento que contiene información estandarizada sobre los principales trastornos psiquiátricos (20). Tiene una concordancia adecuada con otros instrumentos de evaluación de importancia, siendo la ventaja de éste, el ser de fácil aplicación y que no necesita de un entrenamiento riguroso como otros instrumentos (21). Ha sido adaptado lingüísticamente en el EESMLMC del que se basa la muestra de este estudio a través de un ensayo piloto en residentes de 25 viviendas de cada cono de Lima Metropolitana y Callao para un total de 100 viviendas (22).

- Eventos adversos de vida antes de los 18 años: En consenso de un equipo de expertos de INSM HD - HN se consideró de importancia en el contexto del país incluir 8 situaciones de eventos de vida adversos relativos a amenazas y condiciones de vida determinadas por otras conductas de los padres.

- Datos demográficos e indicadores de pobreza: Se incluyeron variables para el cálculo de la pobreza según la metodología de las necesidades básicas insatisfechas (NBI) como características de la vivienda, entre otros (23), siendo la presencia de dos o más NBI correspondiente a familias en pobreza 
Eventos de vida en la infancia y su relación con el trastorno de estrés postraumático en adultos en Lima Metropolitana.

extrema, una NBI a familias pobres y la ausencia de NBI a familias no pobres. Además de preguntas de la Encuesta Nacional de Hogares (ENAHO) 2000 sobre edad, sexo, estado civil, nivel educativo, situación laboral, y sistema de salud adscrito (24).

\section{Aspectos éticos del estudio}

El estudio original tuvo la aprobación del Comité de Ética del Instituto Nacional de Salud Mental "Honorio Delgado - Hideyo Noguchi" y contó con la firma de los consentimientos informados de las personas entrevistadas. El presente estudio fue presentado al referido comité para exoneración de revisión por tratarse de un estudio que realiza un análisis secundario de una base de datos, de igual manera, fue presentado para su revisión y aprobación por el Comité Institucional de Ética e Investigación de la Universidad Peruana Cayetano Heredia. La base datos utilizada para este estudio estuvo anonimizada.

\section{Plan de análisis}

Las prevalencias fueron sopesadas para tener en cuenta la restauración de la distribución de la población en función al sexo y grupos etarios, con intervalos de confianza al 95\% y tomando en consideración el diseño de muestras complejas, así como la probabilidad conocida de selección. En cuanto a las variables, se consideraron los estilos de crianza, los eventos de vida antes de los 18 años y las variables sociodemográficas como variables independientes. En cuanto al análisis bivariados entre las variables, se realizaron pruebas $\mathrm{chi}^{2}$ convertidas al estadístico $\mathrm{F}$ como una variante del estadístico de chi-cuadrado corregido de RaoScott de segundo orden y la significación basada en sus grados de libertad y un nivel de significancia de $<0,05$. Por otro lado, los análisis de asociación se realizaron con cada pregunta por separado así como para las dimensiones recategorizadas. Para los análisis multivariados, se usó la regresión logística multinomial para muestras complejas con la variable de TEPT de por vida y anual como variable dependiente y utilizando la categoría ninguna como referencia. Con todo esto se obtuvieron odds ratios ajustados (OR) para evaluar la asociación entre TEPT de por vida y anual con las variables de estilos de crianza y eventos adversos antes de los 18 años. Las variables sociodemográficas y socioeconómicas fueron ajustadas con sí mismas. Así también, los estilos de crianza fueron controlados con los demás estilos de crianza y de la misma forma los eventos adversos. Ya como último, una vez identificadas las variables con asociaciones estadísticamente significativas en los análisis controlados, se procedió al análisis de un modelo final con aquellas variables con un nivel de significancia de al menos 0,05 . Se utilizó el paquete estadístico de SPSS-V20.

\section{RESULTADOS}

Un total de 4445 adultos fueron entrevistados en el estudio primario. Todos los análisis presentados utilizan la muestra ponderada. La muestra fue distribuida en 48,6\% (IC95\% 46,2-51) varones y $51,4 \%$ (IC95\% 49-53,8) mujeres; y la edad promedio fue de 42,56 años, la cual estuvo comprendida entre los 18 a 94 años. Asimismo, las prevalencias de las variables sociodemográficas y socioeconómicas en la población general, las personas con y sin TEPT se pueden ver en la tabla 1.

Los análisis bivariados entre el TEPT y los factores sociodemográficos solo mostraron asociación significativa con el sexo; el sexo femenino tenía una chance de 2,19 veces de haber padecido TEPT que el masculino (IC95\% 1,49-3,21, p<0,001). En cuanto al análisis multivariado ajustado a todas variables sociodemográficas y socioeconómicas, el sexo femenino mantuvo asociación estadística con el TEPT con un OR de 2,36 (IC95\% 1,56-3,55, p<0,001). El resto de variables sociodemográfica y socioeconómicas no mostraron asociación significativa con el TEPT ni en el análisis bivariado ni el multivariado (tabla 2).

Con respecto a la relación entre los estilos de crianza y el TEPT, los análisis bivariados encontraron asociación estadísticamente significativa con todos los estilos afectivos negativos, la ausencia de todas las variables de estilos afectivos positivos excepto "Le demostraban que estaban interesados en que usted tenga buenas calificaciones", las variables "Le permitían hacer cosas que a sus hermanos(as) no les permitían" y "La ansiedad de sus padres de que algo malo podía sucederle era exagerada" de favoritismo y sobreprotección respectivamente. En cuanto a los análisis multivariados ajustado con las variables dentro del consolidado que corresponden, se encontró que, en los estilos afectivos negativos, las variables "Le dieron más castigos de lo que merecía" y "Le controlaban, pero no le daban afecto" estuvieron asociadas significativamente, con un OR de 1,71 (IC95\% 1,11$2,63, \mathrm{p}=0,016)$ y $2,09(\mathrm{IC} 95 \% 1,30-3,35, \mathrm{p}=0,002)$ respectivamente. Mientras que, en la ausencia de los estilos afectivos positivos, solo la variable "Existía amor y ternura entre usted y sus padres" se encontraba 
Portella C, et al.

Tabla 1. Características sociodemográficas y socioeconómicas de la población adulta de Lima Metropolitana. EESM Lima Metropolitana y Callao 2012.

\begin{tabular}{|c|c|c|c|c|}
\hline \multirow{2}{*}{ Características } & \multirow{2}{*}{$\mathbf{n}$} & \multirow{2}{*}{$\%$} & \multirow{2}{*}{$\begin{array}{l}\text { Con TEPT } \\
\%(\text { IC95\%) }\end{array}$} & \multirow{2}{*}{$\begin{array}{l}\text { Sin TEPT } \\
\%(\text { IC95\%) }\end{array}$} \\
\hline & & & & \\
\hline \multicolumn{5}{|l|}{ Edad (años) } \\
\hline De 18 a 24 & 590 & 17,81 & $4,11(2,71-6,18)$ & $95,89(93,82-97,29)$ \\
\hline De 25 a 44 & 1734 & 42,16 & $5,17(4,01-6,65)$ & $94,83(93,35-95,99)$ \\
\hline De 45 a 64 & 1004 & 27,30 & $5,33(3,76-7,52)$ & $94,67(92,48-96,24)$ \\
\hline De 65 a más & 481 & 12,73 & $5,90(3,95-8,74)$ & $94,10(91,26-96,05)$ \\
\hline \multicolumn{5}{|l|}{ Sexo } \\
\hline Femenino & 2163 & 51,41 & $6,88(5,62-8,40)$ & $93,12(91,60-94,38)$ \\
\hline Masculino & 1646 & 48,59 & $3,26(2,41-4,40)$ & $96,74(95,60-97,59)$ \\
\hline \multicolumn{5}{|l|}{ Lugar de Nacimiento } \\
\hline Lima Metropolitana & 2046 & 57,58 & $5,06(4,02-6,36)$ & $94,94(93,64-95,98)$ \\
\hline Costa & 377 & 9,42 & $5,38(3,35-8,55)$ & $94,62(91,45-96,65)$ \\
\hline Sierra & 1246 & 29,51 & $5,22(3,94-6,87)$ & $94,78(93,13-96,06)$ \\
\hline Selva & 140 & 3,50 & $4,56(1,52-12,85)$ & $95,44(87,15-98,48)$ \\
\hline \multicolumn{5}{|l|}{ Tiempo de Residencia } \\
\hline De 5 a más años & 3622 & 95,58 & $5,10(4,30-6,04)$ & $94,90(93,96-95,70)$ \\
\hline Menor de 5 años & 187 & 4,42 & $5,58(2,27-13,09)$ & $94,42(86,91-97,73)$ \\
\hline \multicolumn{5}{|l|}{ Lengua Materna } \\
\hline Quechua / Aymara & 370 & 8,53 & $6,14(3,99-9,34)$ & $93,86(90,66-96,01)$ \\
\hline Castellano & 3439 & 91,47 & $5,02(4,19-6,01)$ & $94,98(93,99-95,81)$ \\
\hline \multicolumn{5}{|l|}{ Nivel educativo } \\
\hline Primaria o menos & 559 & 13,81 & $4,83(3,21-7,20)$ & $95,17(92,80-96,79)$ \\
\hline Secundaria & 1784 & 45,42 & $5,59(4,45-7,01)$ & $94,41(92,99-95,55)$ \\
\hline Superior no Universitaria & 738 & 20,62 & $4,14(2,82-6,03)$ & $95,86(93,97-97,18)$ \\
\hline Superior Universitaria & 728 & 20,15 & $5,26(3,45-7,94)$ & $94,74(92,06-96,55)$ \\
\hline \multicolumn{5}{|l|}{ Sabe Leer y escribir } \\
\hline No & 78 & 1,94 & $5,33(1,93-13,86)$ & $94,67(86,14-98,07)$ \\
\hline Sí & 3731 & 98,06 & $5,12(4,31-6,06)$ & $94,88(93,94-95,67)$ \\
\hline \multicolumn{5}{|l|}{ Estado Civil } \\
\hline Soltera & 844 & 27,09 & $3,96(2,76-5,66)$ & $96,04(94,34-97,24)$ \\
\hline Casada & 1086 & 31,39 & $5,95(4,44-7,94)$ & $94,05(92,06-95,56)$ \\
\hline Conviviente & 1075 & 25,39 & $5,32(4,06-6,94)$ & $94,68(93,06-95,94)$ \\
\hline $\begin{array}{l}\text { Separado(a)/divorciado(a)/ } \\
\text { viudo(a) }\end{array}$ & 804 & 16,14 & $5,12(3,54-7,37)$ & $94,88(92,06-96,56)$ \\
\hline \multicolumn{5}{|l|}{ ¿Tuvo trabajo? } \\
\hline No & 1337 & 34,81 & $6,17(4,80-7,90)$ & $93,83(92,10-95,20)$ \\
\hline Sí & 2472 & 65,19 & $4,56(3,70-5,61)$ & $95,44(94,39-96,30)$ \\
\hline \multicolumn{5}{|l|}{ Niveles de pobreza } \\
\hline Pobre extremo & 224 & 4,30 & $7,21(4,21-12,07)$ & $92,79(87,93-95,79)$ \\
\hline Pobre & 726 & 17,05 & $6,42(4,61-8,86)$ & $93,58(91,14-95,39)$ \\
\hline No Pobre & 2859 & 78,64 & $4,72(3,84-5,80)$ & $95,28(94,20-96,16)$ \\
\hline
\end{tabular}

TEPT: Trastorno de estrés postraumático, IC: Intervalo de confianza 
Eventos de vida en la infancia y su relación con el trastorno de estrés postraumático en adultos en Lima Metropolitana.

Tabla 2. Características sociodemográficas y socioeconómicas asociadas al trastorno de estrés postraumático en la población adulta de Lima Metropolitana. EESM Lima Metropolitana y Callao 2012.

\begin{tabular}{|c|c|c|c|c|}
\hline \multirow[b]{2}{*}{ Características } & \multicolumn{2}{|c|}{ Análisis Bivariado } & \multicolumn{2}{|c|}{ Análisis multivariado ${ }^{\mathrm{b} /}$} \\
\hline & $\begin{array}{ll}\mathbf{p} & \text { valor } \\
\mathbf{a} / & \end{array}$ & $\mathrm{OR}_{\text {crudo }}(\mathrm{IC} 95 \%)$ & $\begin{array}{l}\mathbf{p} \\
\text { valor }\end{array}$ & OR ${ }_{\text {ajustado }}(\mathrm{IC} 95 \%)$ \\
\hline \multicolumn{5}{|l|}{ Edad (años) } \\
\hline De 18 a 24 & \multirow{4}{*}{0,683} & $1,46(0,81-2,66)$ & 0,100 & $1,90(0,88-4,09)$ \\
\hline De 25 a 44 & & $1,15(0,70-1,90)$ & 0,177 & $1,52(0,83-2,78)$ \\
\hline De 45 a 64 & & $1,11(0,63-1,96)$ & 0,356 & $1,34(0,72-2,49)$ \\
\hline De 65 a más & & 1,00 & & 1,00 \\
\hline \multicolumn{5}{|l|}{ Sexo } \\
\hline Femenino & \multirow{2}{*}{0,000} & $2,19(1,49-3,21)$ & 0,000 & $2,36(1,56-3,55)$ \\
\hline Masculino & & 1,00 & & 1,00 \\
\hline \multicolumn{5}{|l|}{ Lugar de Nacimiento } \\
\hline Lima Metropolitana & \multirow{4}{*}{0,988} & $0,90(0,28-2,84)$ & 0,613 & $0,75(0,24-2,33)$ \\
\hline Costa & & $0,84(0,25-2,87)$ & 0,685 & $0,77(0,22-2,68)$ \\
\hline Sierra & & $0,87(0,27-2,79)$ & 0,811 & $0,86(0,26-2,87)$ \\
\hline Selva & & 1,00 & & 1,00 \\
\hline \multicolumn{5}{|l|}{ Tiempo de Residencia } \\
\hline De 5 a más años & \multirow{2}{*}{0,845} & $1,10(0,42-2,86)$ & 0,711 & $1,21(0,45-3,25)$ \\
\hline Menor de 5 años & & 1,00 & & 1,00 \\
\hline \multicolumn{5}{|l|}{ Lengua Materna } \\
\hline Quechua / Aymara & \multirow{2}{*}{0,401} & $1,24(0,75-2,03)$ & 0,325 & $0,75(0,42-1,33)$ \\
\hline Castellano & & 1,00 & & 1,00 \\
\hline \multicolumn{5}{|l|}{ Nivel educativo } \\
\hline Primaria o menos & \multirow{4}{*}{0,617} & $1,09(0,59-2,02)$ & 0,138 & $1,69(0,85-3,37)$ \\
\hline Secundaria & & $0,94(0,57-1,54)$ & 0,997 & $0,99(0,61-1,65)$ \\
\hline Superior no Universitaria & & $1,29(0,73-2,27)$ & 0,245 & $1,40(0,79-2,48)$ \\
\hline Superior Universitaria & & 1,00 & & 1,00 \\
\hline \multicolumn{5}{|l|}{ Sabe Leer y escribir } \\
\hline No & \multirow{2}{*}{0,936} & $0,96(0,33-2,80)$ & 0,870 & $1,10(0,34-3,56)$ \\
\hline Sí & & 1,00 & & 1,00 \\
\hline \multicolumn{5}{|l|}{ Estado Civil } \\
\hline Soltera & \multirow{4}{*}{0,300} & $1,31(0,76-2,25)$ & 0,865 & $0,86(0,46-1,62)$ \\
\hline Casada & & $0,85(0,53-1,37)$ & 0,640 & $0,68(0,41-1,13)$ \\
\hline Conviviente & & $0,96(0,59-1,56)$ & 0,412 & $0,82(0,48-1,40)$ \\
\hline Separado(a)/divorciado(a)/viudo(a) & & 1,00 & & 1,00 \\
\hline \multicolumn{5}{|l|}{ ¿Tuvo trabajo? } \\
\hline No & \multirow{2}{*}{0,055} & $0,73(0,52-1,01)$ & 0,631 & $0,91(0,63-1,32)$ \\
\hline Sí & & 1,00 & & 1,00 \\
\hline \multicolumn{5}{|l|}{ Niveles de pobreza } \\
\hline Pobre extremo & \multirow{3}{*}{0,133} & $0,64(0,35-1,18)$ & 0,103 & $0,59(0,31-1,11)$ \\
\hline Pobre & & $0,72(0,48-1,10)$ & 0,070 & $0,67(0,43-1,03)$ \\
\hline No Pobre & & 1,00 & & 1,00 \\
\hline
\end{tabular}

a/: Significancia obtenida con la prueba F corregida para muestras complejas; OR: odds ratio; b/: Ajustado por sexo, edad, lugar de nacimiento, tiempo de residencia, lengua materna, nivel educativo, analfabetismo, estado civil, trabajo remunerado, nivel de pobreza. 
con asociación estadística con un OR de 1,72 (IC95\% 1,07-2,75, p=0,024). En cambio, en los de favoritismo, solo mostró asociación significativa la variable "Le permitían hacer cosas que a sus hermanos(as) no les permitían" con TEPT, con OR de 1,77 (IC95\% 1,04-3,01, $\mathrm{p}=0,036$ ). Por último, las variables en sobreprotección no tuvieron asociación estadística en el análisis multivariado (tabla 3).
Por otro lado, en lo referido a los eventos de vida temprana, en los análisis bivariados, sólo se encontró asociación estadística con "Pérdida personal por separación" en los consolidados incluidos en ellos y todas las variables dentro de los eventos adversos de vida tempranas. Asimismo, en los análisis multivariados ajustado por las variables de los consolidados que corresponden, se mostró asociación

Tabla 3. Estilos de crianza asociados a Trastorno de Estrés Postraumático en la población adulta de Lima Metropolitana. EESM Lima Metropolitana y Callao 2012.

\begin{tabular}{|c|c|c|c|c|c|c|}
\hline \multirow{2}{*}{ Estilos de crianza } & \multirow{2}{*}{$\mathbf{n}$} & \multirow{2}{*}{$\%$} & \multicolumn{2}{|c|}{ Análisis Bivariado } & \multicolumn{2}{|c|}{ Análisis multivariado ${ }^{\text {a/ }}$} \\
\hline & & & p valor & $\mathrm{OR}_{\text {crudo }}(\mathrm{IC} 95 \%)$ & p valor & OR ${ }_{\text {ajustado }}($ IC95\%) \\
\hline \multicolumn{7}{|l|}{ Estilo afecto negativo (control y rechazo) } \\
\hline Le dieron más castigos de los que merecía & 787 & 19,19 & 0,000 & $2,50(1,76-3,54)$ & 0,016 & $1,71(1,11-2,63)$ \\
\hline $\begin{array}{l}\text { Sus padres pensaban que los hombres no } \\
\text { deberían llorar }\end{array}$ & 930 & 23,39 & 0,001 & $1,76(1,26-2,47)$ & 0,191 & $1,28(0,89-1,84)$ \\
\hline $\begin{array}{l}\text { Sus padres preferían a los hijos varones en } \\
\text { desventaja de las hijas mujeres }\end{array}$ & 519 & 12,17 & 0,000 & $2,15(1,45-3,17)$ & 0,068 & $1,47(0,97-2,22)$ \\
\hline Le castigaban severamente por cosas pequeñas & 581 & 13,90 & 0,000 & $2,41(1,70-3,43)$ & 0,535 & $1,16(0,73-1,86)$ \\
\hline Le controlaban pero no le daban afecto & 420 & 9,79 & 0,000 & $3,02(2,02-4,53)$ & 0,002 & $2,09(1,30-3,35)$ \\
\hline \multicolumn{7}{|l|}{$\begin{array}{l}\text { Ausencia de estilo afectivo positivo (calor } \\
\text { emocional) }\end{array}$} \\
\hline $\begin{array}{l}\text { Si las cosas le iban mal, sus padres trataban de } \\
\text { reconfortarlo }\end{array}$ & 829 & 19,66 & 0,003 & $1,72(1,21-2,45)$ & 0,500 & $1,17(0,74-1,83)$ \\
\hline Existía amor y ternura entre usted y sus padres & 464 & 10,42 & 0,000 & $2,25(1,51-3,36)$ & 0,024 & $1,72(1,07-2,75)$ \\
\hline $\begin{array}{l}\text { Sus padres le permitían tomar sus propias } \\
\text { decisiones }\end{array}$ & 2020 & 50,52 & 0,010 & $1,58(1,12-2,23)$ & 0,209 & $1,28(0,87-1,88)$ \\
\hline $\begin{array}{l}\text { Sus padres se interesaban por conocer sus } \\
\text { opiniones }\end{array}$ & 1421 & 35,24 & 0,001 & $1,77(1,26-2,49)$ & 0,073 & $1,43(0,97-2,11)$ \\
\hline $\begin{array}{l}\text { Le demostraban que estaban interesados en que } \\
\text { usted tenga buenas calificaciones }\end{array}$ & 1040 & 25,25 & 0,726 & $1,07(0,74-1,53)$ & 0,147 & $0,74(0,50-1,11)$ \\
\hline Le demostraban que le querían & 469 & 10,94 & 0,013 & $1,70(1,12-2,59)$ & 0,772 & $1,08(0,66-1,75)$ \\
\hline \multicolumn{7}{|l|}{ Favoritismo } \\
\hline $\begin{array}{l}\text { Le permitían hacer cosas que a sus hermanos(as) } \\
\text { no les permitían }\end{array}$ & 489 & 12,73 & 0,029 & $1,63(1,05-2,53)$ & 0,036 & $1,77(1,04-3,01)$ \\
\hline $\begin{array}{l}\text { Le engreían más a usted en comparación con sus } \\
\text { hermanos(as) }\end{array}$ & 593 & 15,67 & 0,769 & $1,07(0,68-1,69)$ & 0,989 & $1,00(0,55-1,85)$ \\
\hline Le favorecían con relación a sus hermanos (as) & 531 & 13,74 & 0,915 & $0,98(0,62-1,54)$ & 0,481 & $0,79(0,40-1,54)$ \\
\hline \multicolumn{7}{|l|}{ Sobreprotección } \\
\hline $\begin{array}{l}\text { Le prohibían hacer cosas que a otros niños(as) sí } \\
\text { les era permitido porque temían que algo malo } \\
\text { le podría ocurrir }\end{array}$ & 1277 & 32,65 & 0,957 & $1,00(0,72-1,42)$ & 0,507 & $0,87(0,59-1,30)$ \\
\hline $\begin{array}{l}\text { Eran muy exigentes con respecto a sus } \\
\text { calificaciones escolares, desempeño deportivo o } \\
\text { actividades similares }\end{array}$ & 1781 & 45,31 & 0,457 & $1,15(0,80-1,64)$ & 0,778 & $1,05(0,74-1,50)$ \\
\hline $\begin{array}{l}\text { La ansiedad de sus padres de que algo malo } \\
\text { podía sucederle era exagerada }\end{array}$ & 839 & 21,61 & 0,028 & $1,54(1.05-2,25)$ & 0,095 & $1,46(0,94-2,28)$ \\
\hline $\begin{array}{l}\text { Sus padres lo(a) cuidaban excesivamente (o } \\
\text { demasiado) para evitar que tenga problemas }\end{array}$ & 952 & 24,19 & 0,156 & $1,34(0,89-2,01)$ & 0,532 & $1,17(0,72-1,90$ \\
\hline
\end{tabular}

a/ Modelos ajustados por cada componente de los consolidados de los estilos de crianza correspondiente, OR: odds ratio 
Eventos de vida en la infancia y su relación con el trastorno de estrés postraumático en adultos en Lima Metropolitana.

Tabla 4. Eventos adversos de vida temprana asociados a Trastorno de Estrés Postraumático en la población adulta de Lima Metropolitana. EESM Lima Metropolitana y Callao 2012.

\begin{tabular}{|c|c|c|c|}
\hline & & Análisis Bivariado & Análisis multivariado b/ \\
\hline Eventos adversos de vida temprana & $\%$ & $\begin{array}{l}\mathrm{p} \\
\text { valor }\end{array} \mathrm{OR}_{\text {crudo }}(\mathrm{IC} 95 \%)$ & $\begin{array}{l}\text { p } \\
\text { valor } \quad \text { OR } \\
\text { ajustado } \\
\text { (IC95\%) }\end{array}$ \\
\hline
\end{tabular}

\section{Alguna Disfuncionalidad familiar}

Cuando era menor de 11 años sus padres discutían mucho

$1181 \quad 30,04 \quad 0,000 \quad 1,97(1,39-2,79) \quad 0,125 \quad 1,41(0,91-2,18)$

Alguno de sus padres llegaba ebrio o borracho a casa con regularidad

$\begin{array}{lllllll}1174 & 28,72 & 0,002 & 1,68(1,22-2,33) & 0,439 & 1,16(0,80-1,67) \\ 803 & 20,23 & 0,001 & 1,84(1,31-2,61) & 0,290 & 1,26(0,82-1,94) \\ 191 & 4,37 & 0,000 & 2,87(1,72-4,79) & 0,061 & 1,72(0,98-3,03) \\ 765 & 19,56 & 0,011 & 1,58(1,11-2,26) & 0,348 & 1,20(0,82-1,75) \\ 92 & 2,18 & 0,000 & 5,35(2,80-10,24) & 0,001 & 3,49(1,65-7,39)\end{array}$

Alguno de sus padres fue infiel en la relación

Alguno de sus padres lo(a) hacía sentir culpable por desacuerdos o discusiones de la familia

Sus padres se separaron o divorciaron

Mientras crecía, su familia vivió en permanente riesgo o amenaza a la integridad física de alguno de sus miembros

\section{Pérdida personal por separación}

Sí / no

Abuso o negligencia antes de los 18 años

Alguno de sus padres lo(a) amenazaba con no quererlo(a)

Alguno de sus padres lo(a) amenazaba con matarlo(a)

Alguno de sus padres lo(a) amenazaba con abandonarlo(a)

Mientras crecía sufrió algún tipo de abuso físico como golpes, puñetes o empujones en forma frecuente

Mientras crecía sufrió algún tipo de abuso sexual como ser objeto (o haber sufrido) de intentos o actos sexuales inapropiados, forzados en contra de su voluntad o bajo amenazas

Mientras crecía recibió insultos, agresiones verbales $u$ ofensas en forma frecuente

Mientras crecía fue objeto (o haber sufrido) de chantajes, manipulaciones o humillaciones en forma frecuente

Mientras crecía se ha encontrado en situaciones en las que se ha sentido abandonado
$1543 \quad 38,05 \quad 0,000 \quad 2,10(1,51-2,92) \quad 0,000 \quad 2,10(1,51-2,92)$

$145 \quad 3,35 \quad 0,000 \quad 3,69(2,11-6,46) \quad 0,264 \quad 1,73(0,66-4,56)$

$38 \quad 0,93 \quad 0,011 \quad 3,24(1,31-8,05) \quad 0,684 \quad 0,80(0,27-2,38)$

$130 \quad 3,19 \quad 0,002 \quad 2,49(1,38-4,48) \quad 0,447 \quad 0,68(0,24-1,86)$

$467 \quad 11,38 \quad 0,000 \quad 2,76(1,86-4,10) \quad 0,713 \quad 0,90(0,53-1,55)$

$4,66 \quad 0,000 \quad 4,50(2,70-7,49) \quad 0,037 \quad 2,12(1,05-4,28)$

$511 \quad 12,62 \quad 0,000 \quad 4,21(2,98-5,95) \quad 0,006 \quad 2,07(1,23-3,47)$

$5,57 \quad 0,000 \quad 6,70(4,43-10,13) \quad 0,002 \quad 2,78(1,47-5,24)$

$8,29 \quad 0,000 \quad 3,24(2,09-5,03) \quad 0,362 \quad 1,33(0,72-2,42)$

b/ Modelos ajustados por cada componente de los consolidados de los eventos adversos de vida temprana correspondiente, OR: odds ratio

estadísticamente significativa en la variable de "Alguna disfuncionalidad familiar", siendo esta la variable "Mientras crecía, su familia vivió en permanente riesgo o amenaza a la integridad física de algunos de sus miembros", con un OR de 3,49 (IC 95\% 1,65$7,39, \mathrm{p}=0,001$ ), asimismo, en el consolidado "Pérdida personal por separación", se encontró asociación estadística, con un OR de 2,10 (IC 95\% 1,51-2,92, 
Tabla 5. Estilos de crianza y eventos adversos de vida temprana con asociación estadísticamente significativa a Trastorno de Estrés Postraumático en la población adulta de Lima Metropolitana. EESM Lima Metropolitana y Callao 2012.

\begin{tabular}{ll}
\hline \multirow{2}{*}{ Factores Asociados } & \multicolumn{2}{l}{ Análisis multivariado ${ }^{\mathrm{c} /}$} \\
\cline { 2 - 2 } & p valor $\quad$ OR $_{\text {ajustado }}($ IC95\%) \\
\hline
\end{tabular}

\section{Favoritismo}

Le permitían hacer cosas que a sus hermanos(as) no les permitían $\quad 0,019 \quad 1,70(1,09-2,65)$

\section{Abuso o negligencia antes de los 18 años}

Mientras crecía fue objeto (o haber sufrido) de chantajes, manipulaciones o humillaciones en forma frecuente

c/ Modelo ajustado por edad y sexo, OR: odds ratio

$\mathrm{p}<0,001$ ), y por último, en "Abuso o negligencia antes de los 18 años", las variables "Mientras crecía sufrió algún tipo de abuso sexual como ser objeto (o haber sufrido) de intentos o actos sexuales inapropiados, forzados en contra de su voluntad o bajo amenazas", "Mientras crecía recibió insultos, agresiones verbales u ofensas en forma frecuente" y "Mientras crecía fue objeto (o haber sufrido) de chantajes, manipulaciones o humillaciones en forma frecuente", tuvieron asociación estadística, siendo sus OR de 2,12 (IC 95\% 1,05-4,28, $\mathrm{p}=0,037$ ), 2,07 (IC 95\% 1,23-3,47, $\mathrm{p}=0,006)$ y 2,78 (IC 95\% 1,47-5,24, $\mathrm{p}=0,002)$, de presentar TEPT respectivamente (tabla 4).

Finalmente, en el modelo final, en cuanto a las variables con asociación estadística en los análisis multivariados descrito previamente, al ajustarlos por edad y sexo sólo se encontró asociación estadística con las variables "Le permitían hacer cosas que a sus hermanos(as) no les permitían", con un OR de 1,70 (IC 95\% 1,09-2,65, p=0,019), y "Mientras crecía fue objeto (o haber sufrido) de chantajes, manipulaciones o humillaciones en forma frecuente", con un OR de 6,22 (IC 95\% 4,11-9,42, p<0,001) (tabla 5).

\section{DISCUSIÓN}

Se muestra evidencia sobre la importancia de los eventos de vida y estilos de crianza que podrían influir en el TEPT. Se encuentra que el sexo femenino tiene predisposición de padecer TEPT, un factor de riesgo que ya se conocía, tal como lo descrito en la revisión sistemática de Brewin et al, en la cual se encontró esta relación en personas civiles (14).

En cuanto a la edad, no se halló asociación estadísticamente significativa con el TEPT, factor que en otras poblaciones sí se encontró asociada en edades más tempranas, tal como el estudio realizado en veteranos de guerra (16); o los realizados en población general que tomaron como factor de riesgo el ser menor de 15 años en población general $(6,7)$. En nuestro estudio, probablemente no se encuentre dicha asociación debido a que se tomó como criterio de inclusión personas de 18 años o más, lo cual difiere con los estudios mencionados que hallan como factor de riesgo ser menor de 15 años.

Los estilos de crianza no solo están relacionados al desarrollo y bienestar de los niños, sino que tienen asociación significativa en las conductas de la adultez temprana (25). Esto se vincula a lo postulado por Salirrosas y Saavedra (Perú) quienes evaluaron la relación entre la percepción de algunos estilos de crianza y el episodio depresivo en el adulto de la costa, donde concluyeron que el favoritismo podría estar relacionado a la presencia de cuadros depresivos asociados a otras patología psiquiátrica y con ideas o actos suicidas (26). Por lo tanto, la relación encontrada entre favoritismo y TEPT podría explicarse a que estar en una condición favorecida no ameritada, impida al beneficiado la capacidad de desarrollar conductas resilientes ante un hecho adverso.

Con respecto a los eventos adversos en la infancia, estos perjudican directamente al niño o a su entorno con consecuencias negativas en la adultez (27), se vio asociación con trastornos mentales en la adultez tales como la depresión y el trastorno de ansiedad social $(28,29)$. En nuestro estudio, se encontró relación, dentro de los eventos adversos en la infancia, entre la variable "Mientras crecía fue objeto (o haber sufrido) de chantajes, manipulaciones o humillaciones en forma frecuente" y el TEPT, hallazgo coherente con 
Eventos de vida en la infancia y su relación con el trastorno de estrés postraumático en adultos en Lima Metropolitana.

la revisión sistemática de Brewin et al que relacionó el maltrato en la infancia con el TEPT (14).

Con respecto a alguna disfuncionalidad familiar, la variable "Mientras crecía, su familia vivió en permanente riesgo o amenaza a la integridad física de algunos de sus miembros", predispone a un mayor riesgo de padecer TEPT, un factor de riesgo también estudiado anteriormente. Por ejemplo, Osofsky en su revisión sistemática, evidenció que la exposición a violencia puede tener un impacto significativo en el desarrollo infantil y uno de los efectos negativos puede ser el TEPT (30), así mismo, Bishop et al (República de Sudáfrica) encontraron que las personas con diagnóstico de TEPT sufrieron con más frecuencia abuso de tipo físico y sexual (29).

Otra variable con asociación estadística fue "Pérdida personal por separación”, la cual hace referencia a la ausencia de una figura paterna y/o tutor, que predispone a un mayor riesgo de padecer TEPT, esto podría ser explicado desde la percepción de rechazo del menor, lo cual es coherente con lo discutido por Salirrosas C y Saavedra J, en donde las personas rechazadas, podrían desarrollar estilos de vida negativos, ya que, como lo plantean, el rechazo es una forma de maltrato psicológico, y como se vio en los resultados, esto podría tener cierta relación con TEPT; así también se podría considerar desde la perspectiva de ausencia prolongada, mediante la percepción de aversión de los padres hacia el hijo, que se ha visto están relacionada con niveles altos de ansiedad y depresión, o desde la percepción de padres esquivos, poco comunicativos, que no brindan afecto, esto se ha encontrado que produce poco desarrollo de habilidades de regulación emocional y vulnerabilidad a trastornos depresivos; por otro lado viéndolo desde la perspectiva de pérdida por muerte, se ha encontrado que en un grupo de niños que se encuentran en duelo por dicha pérdida, existe la probabilidad de desarrollar duelo prolongado y TEPT, sugiriendo así que estos experimentan mayor angustia y discapacidad en diferentes dominios $(26,31,32)$. Además, la variable "Mientras crecía sufrió algún tipo de abuso sexual como ser objeto (o haber sufrido) de intentos o actos sexuales inapropiados, forzados en contra de su voluntad o bajo amenazas" predispone a un mayor riesgo de padecer TEPT. Este hallazgo va en armonía con otras investigaciones, por ejemplo, Suelen de Lima et al (Brasil) encontraron que en su población diagnosticada con TEPT, uno de los abusos más frecuente fue el sexual (33); así como la revisión sistemática de Brewin et al que encontraron que la violación (trauma, abuso infantil) tiene relación con
TEPT y hasta un efecto predictivo (14); así mismo, Astin et al (EEUU) compararon 2 grupos de mujeres, donde se encontró que las mujeres que padecían de TEPT tenían mayor probabilidad de haber presentado historia de abuso sexual en la niñez que las que no (34); de igual forma, Copeland et al (EEUU) concluyeron que traumas vinculados con violencia y abuso sexual se asociaron con la tasa más alta de síntomas de TEPT (35).

En cuanto al abuso y negligencia antes de los 18 años, la variable "Mientras crecía recibió insultos, agresiones verbales u ofensas en forma frecuente" predispone a un mayor riesgo de padecer TEPT, este dato es concordante con el estudio de Suelen de Lima et al que encontraron que de los diagnosticados de TEPT en su población, el abuso más frecuente en la infancia que sufrieron fue el emocional (33); por último, la variable "Mientras crecía fue objeto (o haber sufrido) de chantajes, manipulaciones o humillaciones en forma frecuente", predispone a un mayor riesgo de padecer TEPT, esto va de la mano con lo dicho en el estudio mencionado anteriormente; Bishop et al también concluyeron que el abuso emocional puede tener efectos duraderos y contribuir al TEPT (29).

Dentro de todas estas variables, entre eventos adversos de vida y estilos de crianza que salieron con asociación significativas con el TEPT en la regresión logística multivariada ajustada por edad y sexo, encontramos que solo 2 variables tenían asociación estadísticamente significativa; pese a que estas últimas 2 variables son el resultado final de nuestro estudio, fue menester el discutir los resultados anteriores de la tabla 3 y 4 para no perder así, información valiosa que podría ayudar a otros estudios. Hablando de estas 2 variables, "Le permitían hacer cosas que a sus hermanos(as) no les permitían" y "Mientras crecía fue objeto (o haber sufrido) de chantajes, manipulaciones o humillaciones en forma frecuente", estos resultados pueden ser explicados, como se mencionó anteriormente, debido a que la expresión emocional (abuso emocional) de los padres hacia sus hijos puede contribuir al TEPT (29). Hablando del ajuste, se vio que la edad de inicio del abuso es importante, ya que proporciona un punto de partida para evaluar el impacto en la vida, puesto que, dependiendo de esto se puede tener una mayor o menor probabilidad de presentar TEPT (17), o que el abuso devenga en otro trastorno mental ${ }^{(24)}$, pese a que la variable edad no salió asociada significativamente en nuestro estudio, no excluye que pueda haber relación en otras poblaciones (14), reforzando esta idea, vemos que, a pesar que en otras poblaciones el abuso físico 
y sexual es un predictor fuerte de TEPT, en el análisis ajustado, perdió fuerza en comparación con factores emocionales, en el caso de sexo, sí tuvo asociación como se vio al inicio de la discusión.

Este estudio contiene limitaciones que deben ser tomadas en cuenta. El diagnóstico de TEPT en los encuestados se realizó mediante una escala validada en Perú, pero no ha sido corroborado con un médico psiquiatra; también, la deseabilidad social a las enfermedades mentales y la memoria a largo plazo de eventos ocurridos hace años pueden tener influencia en la respuesta de los entrevistados; asimismo, las personas con TEPT tendrían a recordar más los hechos negativos del pasado a comparación de la población general. Además, en esta investigación no se exploró todos los estilos de crianza posibles y no logra diferenciar si los eventos de vida en la infancia son en sí, el estresor o solo un factor predisponente para presentar el TEPT. Finalmente, al ser nuestro estudio transversal, no es posible asumir causalidad.

En conclusión, existe relación entre el favoritismo junto al abuso o negligencia en la infancia con el TEPT en adultos en Lima Metropolitana. Clara importancia se encuentra en haber gozado de preferencias paternas o de los tutores en comparación con hermanos que tuviese, que se puede encontrar este estilo de crianza asociada al TEPT. Del mismo modo, se encuentra que haber sido violentado psicológicamente ya sea con chantajes, manipulaciones o humillaciones en la infancia, predispone a presentar TEPT.

\section{Correspondencia}

Christian Portella Portella

Dirección: Alameda de los Virreyes 450. La Molina, Lima, Perú

Teléfono: 51997562460

Correo electrónico: christian.portella@upch.pe

\section{REFERENCIAS BIBLIOGRÁFICAS}

1. Organización Mundial de la Salud. Clasificación internacional de enfermedades (CIE-10). Ginebra: Organización Mundial de la Salud; 1990. (Consultado el 15 de Enero del 2020). Disponible en: www.who. int/classifications/icd/en/

2. SareenJ.Posttraumatic stressdisorderinadults:impact, comorbidity, risk factors and treatment. Can J Psychiatry. 2014; 59(9):460-7. Doi: 10.1177/070674371405900902

3. Kessler R, Chiu W, Demler O, et al. Prevalence, severity, and comorbidity of 12-month DSMIV disorders in the National Comorbidity Survey
Replication. Arch Gen Psychiatry. 2005; 62(6): $617-$ 27. Doi: $10.1001 /$ archpsyc.62.6.617

4. Van Ameringen M, Mancini C, Pipe B, et al. Posttraumatic stress disorder in Canada. CNS Neuroscience \& Therapeutics. 2008; 14(3): 171-81. Doi: 10.1111/j.1755-5949.2008.00049.x

5. Darves-Bornoz J, Alonso J, de Girolamo G, et al. Main traumatic events in Europe: PTSD in the European study of the epidemiology of mental disorders survey. J Trauma Stress. 2008; 21(5): 45562. doi: 10.1002/jts.20357

6. Norris F, Murphy A, Baker C, Perilla J, Rodriguez F, et al. Epidemiology of trauma and posttraumatic stress disorder in Mexico. J Abnormal Psychology. 2003; 112(4): 646. doi: 10.1037/0021-843x.112.4.646

7. Pérez C, Vicente B, Zlotnick C, Kohn R, Johnson J, et al. Estudio epidemiológico de sucesos traumáticos, trastorno de estrés post-traumático y otros trastornos psiquiátricos en una muestra representativa de Chile. Salud Ment (Mex). 2009; 32(2): 145-153. (Consultado el 15 de Enero del 2020). Disponible en: www.ncbi. nlm.nih.gov/pmc/articles/PMC2990643/

8. Instituto Nacional de Salud Mental. Estudio Epidemiológico de Salud Mental en Lima Metropolitana y Callao - Replicación 2012 Informe General. Anales de Salud Mental. 2013; 29 (Suplemento1). (Consultado el 15 de Enero del 2020). Disponible en: www.insm.gob.pe/ investigacion/archivos/estudios/2012\%20ASM\%20 -EESM\%20-LM.pdf

9. Instituto Nacional de Salud Mental. Estudio epidemiológico de salud mental en la selva rural 2009. Informe general. Anales de salud mental. 2012; 28 (Suplemento 2). (Consultado el 15 de Enero del 2020). Disponible en: www.insm.gob.pe/ investigacion/archivos/estudios/2009-ASM-EESMSR.pdf

10. Varela E, Hensley-Maloney L. The influence of culture on anxiety in Latino youth: a review. Clin Child Fam Psychol Rev. 2009; 12: 217-33. doi: 10.1007/s10567-009-0044-5

11. Cairo J, Dutta S, et al. The Prevalence of Posttraumatic Stress Disorder Among Adult Earthquake Survivors in Peru. Rev Disaster Medicine and Public Health Preparedness. 2010; 4(1): 39-46. doi:10.1017/S1935789300002408

12. Suarez E. Two decades later: the resilience and posttraumatic response of indigenous Quechua girls and adolescents in the aftermath of the Peruvian armed conflict. Rev Child Abuse \& Neglect. 2013; 23(2): 200-10. doi: 10.1016/j.chiabu.2012.09.011

13. Ozer E, Best S, Lipsey T,Weiss D. Predictors of posttraumatic stress disorder and symptoms in adults: A meta-analysis. Psychological Bulletin. 2003; 129(1): 52-73. doi: 10.1037/0033-2909.129.1.52

14. Brewin C, Andrews B, Valentine J. Meta-analysis of 
Eventos de vida en la infancia y su relación con el trastorno de estrés postraumático en adultos en Lima Metropolitana.

risk factors for posttraumatic stress disorder in trauma-exposed adults. J Consult Clin Psychol. 2000; 68(5): 748-66. doi: 10.1037/0022-006X.68.5.748

15. UNICEF. Definición de la infancia. UNICEF; 2005. (Consultado el 15 de febrero del 2021). Disponible en: www.unicef.org/sowc05/spanish/childhoodde fined.html

16. Bremner J, Southwick S, Johnson D, Yehuda R, Charney D. Childhood physical abuse and combatrelated posttraumatic stress disorder in Vietnam veterans. Am J Psychiatry. 1993; 150(2): 235-9. Doi: 10.1176/ajp.150.2.235

17. Breslau N, Chilcoat H, Kessler R, Davis G. Previous exposure to trauma and PTSD effects of subsequent trauma: results from the Detroit area survey of trauma. Am J Psychiatry. 1999; 156(6): 902-7. Doi: 10.1176/ ajp.156.6.902

18. Instituto Nacional de Salud Mental. Confiabilidad y validez de los cuestionarios de salud mental de Lima y de la Selva Peruana. Anales de Salud Mental. 2009; 25(1): 1-259. (Consultado el 24 de marzo del 2020). Disponible en: www.insm.gob.pe/ojsinsm/index.php/ Revista1/article/view/97

19. Someya T, Uehara T, Kadowaki M, et al. Factor analysis of the EMBU scale in a large sample of Japanese volunteers. Acta Psychiatr Scand. 1999; 100(4):252-7. Doi: 10.1111/j.1600-0447.1999. tb10858.X

20. Lecrubier Y, Weiller E, Hergueta T, et al. Mini International Neuropsychiatric Interview - Versión en español 5.0.0. Madrid: Instituto IAP; 2000.

21. Sheehan DV, Lecrubier Y, Sheehan KH, et al. The Mini-International Neuropsychiatric Interview (M.I.N.I.): The Development and Validation of a Structured Diagnostic Psychiatric Interview for DSM-IV and ICD-10. J Clin Psychiatry. 1998; 59(20): 22-33.

22. Instituto Nacional de Salud Mental. Estudio Epidemiológico Metropolitano en Salud Mental 2002 - Informe General. Anales de Salud Mental. 2002; 18 (Suplemento 1 y 2). (Consultado el 24 de marzo del 2020). Disponible en: www.insm.gob.pe/ investigacion/archivos/estudios/2002-ASMEESM-M/files/res/downloads/book.pdf

23. Feres JC, Macero X. Enfoques para la medición de la pobreza: Breve revisión de la literatura. Santiago de Chile: UN; 2001. (Consultado el 24 de marzo del 2020). Disponible en: www.researchgate. net/publication/239928852_Enfoques_para_la medicion_de_la_pobreza_Breve_revision_de_la_ literatura

24. Instituto Nacional de Estadística e Informática. Principales resultados de la Encuesta Nacional de Hogares sobre Condiciones de Vida y Pobreza (ENAHO) - IV Trimestre 2001. Lima: Instituto Nacional de Estadística e Informática; 2002.
(Consultado el 24 de marzo del 2020). Disponible en: www.inei.gob.pe/media/MenuRecursivo/ boletines/4346.pdf

25. Darling N, Steinberg L. Parenting style as context: An integrative model. Psychol.Bull. 1993; 113(3):487-96. doi: 10.1037/0033-2909.113.3.487

26. Salirrosas C, Saavedra J. Percepción de algunos estilos de crianza y el episodio depresivo en el adulto. Rev Neuropsiquiatr. 2014; 77(3):160-7. Doi: 10.20453/rnp.v77i3.2030.

27. Lester S, Lorenc T, Sutcliffe K, et al. What helps to support people affected by Adverse Childhood Experiences? A Review of Evidence. London: EPPICentre; 2019. (Consultado el 24 de marzo del 2020). Disponible en: eppi.ioe.ac.uk/CMS/Portals/0/ACEs_ report-041119_1.pdf

28. Rudenstine S, Cohen G. Eventos adversos de la infancia y el riesgo de depresión de nuevo inicio y trastorno de estrés postraumático entre los soldados de la Guardia Nacional de EE. UU. Rev Medicina militar. 2015; 180(9): 972-8. Doi:10.7205/ MILMED-D-14-00626

29. Bishop M, Rosenstein D. An analysis of early developmental trauma in social anxiety disorder and posttraumatic stress disorder. Ann Gen Psychiatry. 2014; 13(16): 1-14. Doi:10.1186/1744-859X-13-16

30. Osofsky J. The impact of violence on children. Rev The future of children. 1999; 9(3): 33-49. Doi: $10.2307 / 1602780$

31. Yap M, Pilkington P, Ryan S, Jorm A. Parental factors associated with depression and anxiety in young people: A systematic review and meta-analysis. J. Affect. Disord. 2014; 156:8-23. Doi: 10.1016/j. jad.2013.11.007

32. Boelen oelSpuij puiReijntjes eijntjes. jad.2013.11.007rg/10.1016/j.jad.2013.11.007» \t 《_ blank» g people: A systematic rePsychiatry Res. 2017; 258:518-24. Doi: 10.1016/j.psychres. 2017.09.002

33. Suelen de Lima B, Acosta M, et al. Suicide risk and childhood trauma in individuals diagnosed with posttraumatic stress disorder. Rev Tendencias Psiquiatría Psicoterapeuta. 2018. 40(3): 253-7. doi: 10.1590/2237-6089-2017-0101

34. Astin M, Ogland-Hand S, Coleman E, et al. Posttraumatic stress disorder and childhood abuse in battered women: Comparisons with maritally distressed women. J Consult Clin Psychol. 1995; 63(2): 308-12. doi: 10.1037/0022-006X.63.2.308

35. Copeland W, Keeler G, Anglod A, Costello E. Traumatic events and posttraumatic stress in childhood. Arch Gen Psychiatry. 2007; 64(5): 57784. Doi:10.1001/archpsyc.64.5.577

Recibido: 10/06/2021

Aceptado: 13/12/2021 
Anexos

Definición operacional de variables

Variables dependientes

\begin{tabular}{|c|c|c|c|}
\hline Variable & $\begin{array}{lll}\text { Tipo y escala de } \\
\text { variable }\end{array}$ & Definición operacional & Codificación \\
\hline \multicolumn{4}{|c|}{ Indicadores de trastornos psiquiátricos } \\
\hline $\begin{array}{l}\text { TEPT de } \\
\text { por vida }\end{array}$ & Categórica Nominal & $\begin{array}{l}\text { Uso de encuesta MINI en el que se incluyen criterios para TEPT de } \\
\text { acuerdo a definición de CIE-10, y que además sintomatología persista } \\
\text { más de un año después del trauma hasta la actualidad }\end{array}$ & $\begin{array}{l}0: \text { No } \\
\text { 1: Sí }\end{array}$ \\
\hline TEPT anual & Categórica Nominal & $\begin{array}{l}\text { Uso de encuesta MINI en el que se incluyen criterios para TEPT de } \\
\text { acuerdo a definición de CIE-10, y que además sintomatología persista } \\
\text { hasta un año después del trauma }\end{array}$ & $\begin{array}{l}0: \text { No } \\
\text { 1: Sí }\end{array}$ \\
\hline
\end{tabular}

\section{Variables independientes}

\begin{tabular}{|c|c|c|c|}
\hline Variable & $\begin{array}{l}\text { Tipo y escala } \\
\text { de variable }\end{array}$ & Definición operacional & Codificación \\
\hline \multicolumn{4}{|c|}{ Variables sociodemográficas } \\
\hline Sexo & $\begin{array}{l}\text { Cualitativa } \\
\text { dicotómica }\end{array}$ & $\begin{array}{l}\text { Describe el género de una persona } \\
\text { 1: Masculino }\end{array}$ & 0: Femenino \\
\hline Edad & $\begin{array}{l}\text { Cuantitativo } \\
\text { discreto } \\
\text { ordinal }\end{array}$ & $\begin{array}{l}\text { Tiempo transcurrido desde el nacimiento hasta la actualidad } \\
\text { 2: } 25 \text { - } 44 \text { años } \\
\text { 3: } 45 \text { - } 64 \text { años } \\
\text { 4: } 65 \text { o más }\end{array}$ & 1: $18-24$ años \\
\hline Estado civil & $\begin{array}{l}\text { Cualitativa } \\
\text { discreta } \\
\text { nominal }\end{array}$ & $\begin{array}{l}\text { Conjunto de condiciones o cualidades jurídicamente relevantes que } \\
\text { tiene una persona frente a una familia determinada } \\
\text { 1: Casado } \\
\text { 2: Separado } \\
\text { 3: Divorciado } \\
\text { 4: Viudo }\end{array}$ & 0: Soltero \\
\hline Nivel de instrucción & $\begin{array}{l}\text { Cualitativa } \\
\text { discreta } \\
\text { nominal }\end{array}$ & $\begin{array}{l}\text { Grado de instrucción o educación media } \\
\text { 1: Secundaria } \\
\text { 2: Superior no universitario } \\
\text { 3: Superior universitario }\end{array}$ & $\begin{array}{l}0: \text { Sin nivel/ } \\
\text { primaria }\end{array}$ \\
\hline
\end{tabular}

Estado socioeconómico

\begin{tabular}{|c|c|c|c|c|}
\hline Nivel de pobreza & $\begin{array}{l}\text { Medida a } \\
\text { través de las } \\
\text { Necesidades } \\
\text { básicas } \\
\text { insatisfechas }\end{array}$ & \multicolumn{2}{|c|}{$\begin{array}{l}\text { Es pobre aquel hogar que, dadas sus fuentes de bienestar, no puede } \\
\text { satisfacer sus necesidades, por más eficientemente que las use. } \\
\text { 1: Pobre } \\
\text { 2: Pobre extremo }\end{array}$} & 0: No pobre \\
\hline $\begin{array}{l}\text { Trabajo en la última } \\
\text { semana }\end{array}$ & $\begin{array}{l}\text { Cualitativa } \\
\text { dicotómica }\end{array}$ & \multirow{2}{*}{\multicolumn{2}{|c|}{$\begin{array}{l}\text { Trabajo remunerado que realizó en la última semana } \\
\text { 1: Sí }\end{array}$}} & $0:$ No \\
\hline Estilos de crianza & & & & \\
\hline \multirow{5}{*}{$\begin{array}{l}\text { Estilo afectivo } \\
\text { negativo (control y } \\
\text { rechazo) }\end{array}$} & \multirow{5}{*}{$\begin{array}{l}\text { Cualitativa } \\
\text { Nominal }\end{array}$} & \multirow{5}{*}{$\begin{array}{l}\text { Presencia de } \\
\text { los siguientes } \\
\text { estilos de } \\
\text { crianza } \\
\text { relacionados }\end{array}$} & Le dieron más castigos de los que merecía & 0: No, 1: Sí \\
\hline & & & $\begin{array}{l}\text { Lo/la castigaban severamente (o mucho) por cosas } \\
\text { pequeñas }\end{array}$ & 0: No, 1: Sí \\
\hline & & & Lo/la controlaban, pero no le daban afecto & 0: No, 1: Sí \\
\hline & & & $\begin{array}{l}\text { Sus padres pensaban que los hijos hombres no } \\
\text { deberían llorar }\end{array}$ & 0: No, 1: Sí \\
\hline & & & $\begin{array}{l}\text { Sus padres preferían a los hijos varones en } \\
\text { comparación con las hijas mujeres }\end{array}$ & 0: No, 1: Sí \\
\hline
\end{tabular}


Eventos de vida en la infancia y su relación con el trastorno de estrés postraumático en adultos en Lima Metropolitana.

Ausencia de estilo afectivo positivo (calor emocional) o Nominal autonomía

$\begin{array}{ll}\text { Favoritismo } & \begin{array}{l}\text { Cualitativa } \\ \text { Nominal }\end{array} \\ & \\ \text { Sobreprotección } & \begin{array}{l}\text { Cualitativa } \\ \text { Nominal }\end{array}\end{array}$

Presencia de los siguientes estilos de crianza

relacionados

Presencia de los siguientes estilos de crianza relacionados estilos de crianza relacionados
Si las cosas le iban mal, sus padres trataban de confortarlo(a), animarlo(a) y apoyarlo(a)

Existía amor y ternura entre usted y sus padres

Ausencia de Le demostraban que lo/la querían

Le permitían tomar sus propias decisiones, por ejemplo, elegir su ropa, escoger a sus amistades, los estudios que debía seguir, distracciones, etc.

Le demostraban que estaban interesados en que usted tuviera buenas calificaciones

Sus padres se interesaban por conocer sus opiniones (o ideas)

Le permitían hacer cosas que a sus hermanos(as) no les era permitido

Lo/la engreían más a usted en comparación con sus hermanos(as)

Lo/la favorecían con relación a sus hermanos(as)

Le prohibían hacer cosas que a otros niños(as) sí les

era permitido, porque temían que algo malo le podría 0 : No, 1: Sí ocurrir

Eran muy exigentes con respecto a sus calificaciones escolares, desempeño deportivo o actividades similares

La ansiedad de sus padres de que algo malo podía sucederle era exagerada

Sus padres lo(a) cuidaban excesivamente (o demasiado) para evitar que tenga problema
$0:$ Sí, 1: No

0: Sí, 1: No

0: Sí, 1: No

0: Sí, 1: No

0: Sí, 1: No

0: Sí, 1: No

0: No, 1: Sí

0: No, 1: Sí

0: No, 1: Sí

0: No, 1: Sí

0: No, 1: Sí

0: No, 1: Sí

\section{Eventos adversos de vida}

Alguna

disfuncionalidad familiar

Perdida personal por separación, abandono o muerte
Cualitativa Nominal

Cualitativa Nominal
Presencia de los siguientes eventos adversos relacionados

Presencia de los siguientes eventos adversos relacionados
Cuando era menor de 11 años sus padres discutían mucho

0: No, 1: Sí

Alguno de sus padres llegaba ebrio o borracho a casa con regularidad (o frecuencia)

0: No, 1: Sí

Sus padres se separaron o divorciaron

0: No, 1: Sí

Alguno de sus padres fue infiel en la relación

0: No, 1: Sí

0: No, 1: Sí desacuerdos o discusiones de la familia

Mientras crecía su familia vivió en permanente riesgo o amenaza a la integridad física de alguno de sus miembros

Alguno de sus padres estaba mucho tiempo ausente de casa

0: No, 1: Sí

Perdida del padre antes de los 18 años

0: No, 1: Sí

Perdida del padre antes de los 12 años

0: No, 1: Sí

Pérdida de la madre antes de los 12 años

0: No, 1: Sí

Pérdida de la madre antes de los 18 años
0: No, 1: Sí 
Portella C, et al.

Abuso o negligencia Cualitativa antes de los 18 años Nominal
Presencia de

los siguientes

eventos

adversos

relacionados
Mientras crecía recibió insultos, agresiones verbales $\mathrm{u}$ ofensas en forma frecuente

Mientras crecía, sufrió algún tipo de abuso físico como golpes, puñetes o empujones en forma frecuente

Se ha encontrado en situaciones en las que se ha sentido abandonado(a) porque no le procuraban alimentos, cuidados médicos, ropa adecuada, o afecto

Fue objeto (o haber sufrido) de chantajes, manipulaciones o humillaciones en forma frecuente

Sufrió algún tipo de abuso sexual como ser objeto (o haber sufrido) de intentos o actos sexuales inapropiados, forzados en contra de su voluntad o 0: No, 1: Sí bajo amenazas

Alguno de sus padres lo(a) amenazaba con no quererlo(a)

0: No, 1: Sí

Alguno de sus padres lo(a) amenazaba con abandonarlo(a)

0: No, 1: Sí

0: No, 1: Sí

Alguno de sus padres lo(a) amenazaba con
0: No, 1: Sí

0: No, 1: Sí

0: No, 1: Sí

0: No, 1: Sí matarlo(a) 